\section{P295 PREVALENCE OF STIS AMONG NEPALESE WOMEN POPULATION}

Prasanna Upreti*. Prabhat Kiran Sewa Samaj (NGO), Community Service, Chitwan, Nepal

10.1136/sextrans-2019-sti.413

Background Sexually transmitted infections (STIs) cause considerable morbidity worldwide, especially among women of reproductive age. Data on STI prevalence in Nepal are limited, and prevention and control programs have focused primarily on HIV infection. According to national reports, the estimated prevalence of HIV infection was $0.2-0.3 \%$ in 2013. In high-risk populations, however, there has been observed a concentrated HIV epidemic with an infection prevalence of $1.7-18.3 \%$. So far, data on other STIs from Nepal are mostly derived from hospital or health-camp records or from high-risk populations.

Methods A population-based study of non-pregnant women with age 15 years and above, who were married or had a history of marriage in the past, residing in rural communities in Nepal. Data on sociodemographic characteristics, reproductive history, and genitourinary symptoms were collected, and a gynecological examination was also conducted. Cervical samples were analyzed by real-time PCR for Neisseria gonorrhoeae, Chlamydia trachomatis, and Trichomonas vaginalis and HPV, and a serum sample was analyzed for syphilis, hepatitis $B$ virus (HBV) and HIV infection by serology.

Results Of 3570 eligible women, 73\% participated. Trichomoniasis, Chlamydia trachomatis infection, HPV and HBV infection, and syphilis were detected in 7.2\%, 1.2\%, 11.4\%, 1.7\%, and $1.5 \%$ of the women. None had gonorrhea or HIV infection. Of those with genitourinary symptoms, 9.4\% had a curable STI. Vaginal discharge classified as abnormal by gynecological examination, but not self-reported discharge, was significantly associated with laboratory diagnosis of a curable STI. Risk factors for trichomoniasis were reproductive age and high cast/ethnicity. Due to low prevalence, risk factors for other STIs could not be accurately determined.

Conclusion We observed a high prevalence of HPV infection followed by trichomoniasis, while other STIs were rare among women in rural Nepal. There was no association between genitourinary symptoms and laboratory-confirmed STIs.

Disclosure No significant relationships.

\section{P299 EPIDEMIOLOGY OF SEXUALLY TRANSMITTED INFECTIONS AT GABRIEL TOURE TEACHING HOSPITAL, BAMAKO, MALI}

${ }^{1}$ Ibrahima Téguété* ${ }^{2}$ Fatoumata Korika Tounkara, ${ }^{3}$ Seydou Fane, ${ }^{3}$ Abdoulaye Sissoko, ${ }^{1}$ Youssouf Traore. ${ }^{1}$ Gabriel Toure Teaching Hospital, Bamako, Mali; ${ }^{2}$ Axe Santé des Populations et Pratiques Optimales en Santé, HSS, Social and Preventive Medicine, Québec, Canada; ${ }^{3}$ Gabriel Toure Teaching Hospital, Gynecology and Obstetric, Bamako, Mali

\subsection{6/sextrans-2019-sti.414}

Background Sexually transmitted infections (STIs), including human immunodeficiency virus (HIV) remain a major public health problem in Africa. Indeed, they carried high morbidity and mortality in this region. The study aimed (1) to estimate the prevalence of STIs/genital infections in gynecological admissions at Gabriel Toure Teaching Hospital; (2) assess the association between HIV and STIs/genital infections.

Methods We carried out a cross-sectional study using a gynecological admissions database at Gabriel Toure Teaching Hospital, from January 1st, 2006 to December 31st, 2010. We estimated the prevalence of STIs and genital infections. A multivariate logistic regression model was used to estimate the Adjusted Odds Ratios (aOR) of HIV infection and risk factors with $95 \%$ Confidence Interval $(95 \% \mathrm{CI})$. STIs and genital infections were the main exposure variables.

Results Among 9112 women admitted in a gynecological unit, 2240 women have laboratory test. Of these, STIs (Chlamydia trachomatis and Trichomonas vaginalis) prevalence were $7.8 \%$, genital infection such as candida albicans (CA) represented $40.0 \%$, bacterial vaginosis (G. vaginalis, U. urealiticum and $\mathrm{M}$. hominis), 36.2\%, while E. coli, Enterobacter and Enterococcus represented together $3.8 \%$. STIs prevalence was higher in women aged 20 to 40 years old compared to adolescent $(<20$ years) and older women $(>40), 6.0 \% \%$ vs $1.9 \%$, respectively. A same figure was observed with non-STIs. We noted a higher prevalence of STIs in cervicitis cases $(11.8 \%)$. According to HIV status, CA was the main infection observed with a frequency of $60.0 \%$ in $\mathrm{HIV}+, 51.8 \%$ in HIV- and $36.3 \%$ in undetermined HIV status. The main risk factor associated with HIV infection was polygamous couple compared to monogamy $(\mathrm{aOR}=4.87$; 95\%CI: $1.02-23.22)$.

Conclusion Women aged 20 to 40 years old carry the highest burden of genital infection at Gabriel Toure Teaching Hospital. CA was the most associated with HIV infection.

Disclosure No significant relationships.

\section{P300 NEIGHBORHOOD VIOLENT CRIME AND HIV TRANSMISSION RISK: A TEMPORAL AND SPATIAL EXPLORATION OF THEIR ASSOCIATION}

${ }^{1}$ Aruna Chandran* ${ }^{2}$ Linxuan Wu, ${ }^{3}$ Christina Schumacher, ${ }^{3}$ Errol Fields, ${ }^{4}$ Amanda Long, ${ }^{5}$ Jacky Jennings. 'Johns Hopkins Bloomberg School of Public Health, Epidemiology, Baltimore, USA; ${ }^{2}$ Johns Hopkins School of Medicine, Center for Child and Community Health Research, Baltimore, USA; ${ }^{3}$ Johns Hopkins School of Medicine, Baltimore, USA; ${ }^{4}$ Johns Hopkins University School of Medicine, Baltimore, USA; ${ }^{5}$ Johns Hopkins University School of Medicine, Center for Child and Community Health Research (CCHR), Baltimore, USA

10.1136/sextrans-2019-sti.415

Background Experiencing violence, both exposure and victimization, has been associated with negative health outcomes including increased mental health problems and sexual risk behaviors. This ecological analysis aimed to explore the ecologic relationship between aggregate violent crime and HIV transmission risk at the census tract level, including effects of spatial dependence.

Methods Violent crime data reported with address of the incident by the Baltimore Police Department as well as HIV viral load information for those with reported addresses and a viral load of $>400$ copies $/ \mathrm{mL}$ collected by the Maryland Department of Health and Mental Hygiene were geocoded and aggregated to the census tract level. Community viral load (CVL) was calculated as the prevalence of uncontrolled viral 
load in each tract. A negative binomial regression model was used to test the association between violent crime events and CVL, including spatial lag from shared-boundary census tracts as well as mean age, proportion male, labor force participation, educational attainment, and residential instability as key covariates.

Results The annual violent crime rate in Baltimore City in the 5-year period from 2012-2016 was 29.5 per 1,000 population; rates varied widely between census tracts, ranging from 2 to 189.4 per 1,000 . The mean CVL was 4.27 per 1,000 population, with a range from 0 to 18.8 by census tract. In the adjusted model, a 100-unit increase in violent crimes was associated with a 19\% increase in CVL (RR: 1.19, 95\% CI: $1.13,1.27)$.

Conclusion Our study shows a statistically significant association between violent crime rates and HIV transmission risk in local areas. This study highlights the need for community-level interventions aimed to address effects of violence exposure in order to effectively combat the ongoing HIV epidemic among vulnerable populations in urban settings.

Disclosure No significant relationships.

\section{P302 ACHIEVING THE THIRD 90: KEEPING PREGNANT AND BREASTFEEDING WOMEN LIVING WITH HIV VIRALLY SUPPRESSED IN WESTERN NIGERIA}

${ }^{1}$ Saheed Usman, ${ }^{2}$ biwumi Usman*. ${ }^{1}$ APIN Public Health Intiatives, Abuja, Nigeria; ${ }^{2}$ Kids and Teens Resource Centre, Akure, Nigeria

\subsection{6/sextrans-2019-sti.416}

Background In 2016, Nigeria transitioned to 'Test \& Treat', a policy where all people living with HIV (PLHIV) are treated with lifelong antiretroviral therapy (ART) regardless of clinical or immunological status. Hypothesis tested was antiretroviral therapy adherence effect on viral load outcome. The aim of this study is to assess the viral load suppression rates among HIV positive pregnant \& breastfeeding women in Western Nigeria.

Methods This study was an observational prospective study of pregnant \& breastfeeding HIV positive subjects already initiated on antiretroviral therapy for at least six months, enrolled in health facilities across supported facilities in Western Nigeria, during a 12-month observation period starting October 2016 till September 2017. Quantitative viral load analysis was done using Polymerase Chain Reaction, Roche Cobas Taqman 96 Analyzer.

Results A total of 243 subjects were recruited. The mean age of $32.58 \pm 4.18$ years. 69 are breastfeeding while 174 were pregnant women. 208 (85.6\%) \& 188 (77.4\%) had viral suppression of $<1000 \&<50$ RNA copies per ml respectively. The 35 subjects went through peer counseling by trained PLHIV and enhanced adherence counseling (EAC) for three months and viral load test repeated three further months after, which made 221 (90.9\%) \& 202 (83.1\%) of the subjects have $<1000$ RNA \& $<50$ RNA copies per ml respectively during the observation. The PLHIVs joined the institutionalized social-media driven support group \& decentralized care model ensuring they achieve the third 90 at undetectable level. ART adherence has significant effect on viral load outcome $\left(\chi^{2}=5.86\right.$, df $\left.=1, \mathrm{P}=0.001\right)$.

Conclusion ART adherence counseling is key to the achieving viral suppression and determine infection prognosis, thus, developing robust continuous quality improvement (CQI) plans to address issues across the cascade ultimately helping in the monitoring of HIV/AIDS disease progression and decrease treatment failure tendencies.

Disclosure No significant relationships.

\section{P304 ACHIEVING THE THIRD 90: KEEPING ADOLESCENTS LIVING WITH HIV VIRALLY SUPPRESSED IN RURAL NIGERIA IN THE ERA OF TEST AND TREAT}

Saheed Usman*, Prosper Okonkwo, Oluwatoyin Jolayemi, Jay Osi-Samuels, Patrick Akande, Babatunde Ladi-Akinyemi, Oluremi Olaitan, Femi Owolagba, Matthias Alagi, Eke Ofuche. APIN Public Health Intiatives, Abuja, Nigeria

\subsection{6/sextrans-2019-sti.417}

Background In 2016, Nigeria transitioned to 'Test \& Treat', a policy where all people living with HIV (PLHIV) are treated with lifelong antiretroviral therapy (ART). There are unique challenges achieving viral suppression in ALHIV mainly due to increased stigma \& lack of social support. Hypothesis tested was ART adherence effect on viral load outcome. We examined viral suppression among adolescents living with HIV in Western Nigeria.

Methods This study was an observational prospective cohort study of adolescents living with HIV (ALHIV) already initiated on antiretroviral therapy for at least six months, enrolled in health facilities across supported facilities in Western Nigeria, during a 12-month observation period starting October 2016 till September 2017. Quantitative viral load analysis was done using Polymerase Chain Reaction, Roche Cobas Taqman 96 Analyzer.

Results A total of 126 (64 males \& 62 females) subjects were recruited. The mean age of $13.58 \pm 4.26$ years. $83(65.9 \%)$ $\& 71(56.3 \%)$ had viral suppression of $<1000 \&<50$ RNA copies per $\mathrm{ml}$ respectively. The 43 subjects went through peer counseling by trained ALHIV and enhanced adherence counseling (EAC) for three months and viral load test repeated three further months after, which made 113 (89.7\%) \& 101 (80.1\%) of the subjects have $<1000$ RNA $\&<50$ RNA copies per $\mathrm{ml}$ respectively during the observation. The ALHIVs joined the institutionalized social-media driven support group $\&$ adolescent decentralized care model ensuring they achieve the third 90 at an undetectable level. ART adherence has significant effect on viral load outcome $\left(\chi^{2}=6.42\right.$, df $=1, \mathrm{P}$ $=0.001)$.

Conclusion ART adherence counseling is key to the achieving viral suppression and determine infection prognosis, thus, developing robust continuous quality improvement (CQI) plans to address issues across the cascade ultimately helping in the monitoring of HIV/AIDS disease progression and decrease treatment failure tendencies.

Disclosure No significant relationships. 